\title{
Second Primary Malignancies in Patients with Well-differentiated/Dedifferentiated Liposarcoma
}

\author{
ERIC JUNG ${ }^{1}$, MARCO FIORE ${ }^{2}$, ALESSANDRO GRONCHI ${ }^{2}$, VALERIE GRIGNOL ${ }^{3}$, RAPHAEL E. POLLOCK ${ }^{3}$, \\ SUSAN S. CHONG ${ }^{1}$, SHEFALI CHOPRA ${ }^{4}$, ANN S. HAMILTON ${ }^{5}$ and WILLIAM W. TSENG ${ }^{1}$ \\ Departments of ${ }^{1}$ Surgery, and ${ }^{5}$ Preventive Medicine, University of Southern California, \\ Keck School of Medicine, Los Angeles, CA, U.S.A.; \\ ${ }^{2}$ Department of Surgery, IRCCS Foundation National Tumor Institute, Milan, Italy; \\ ${ }^{3}$ Department of Surgery, The James Comprehensive Cancer Center, Ohio State University, Columbus, OH, U.S.A.; \\ ${ }^{4}$ Discovery Research Program, Department of Pathology and Laboratory Medicine, \\ University of Southern California, Los Angeles, CA, U.S.A.
}

\begin{abstract}
Background: Well-differentiated/dedifferentiated $(W D / D D)$ liposarcoma is a rare malignancy of putative adipocyte origin. To our knowledge, there have only been isolated case reports describing second primary cancer in patients with this disease. We report on a combined case series of such patients and explore the frequency of this occurrence using a national cancer database. Materials and Methods: Demographics and clinicopathological data were collected from patients with WD/DD liposarcoma who were found to have a concurrent or subsequent second primary cancer, at one of three sarcoma referral centers from 2014-2016. The Surveillance, Epidemiology and End Results (SEER) database was also queried to identify adult patients diagnosed with WD/DD liposarcoma between 1973-2012. Observed/expected (O/E) ratios of second primary malignancies among these cases were calculated by comparison to the age-adjusted cancer incidence in the general population using SEER* stat software. Results: In total, 26 out of 312 consecutive patients (8.3\%) with WD/DD liposarcoma at our centers had a second primary cancer identified within 2 years of liposarcoma diagnosis. In the SEER database, among 1,845 patients with WD/DD liposarcoma, 75 (4.1\%) had a second cancer within 2 years after liposarcoma diagnosis $($ O/E ratio $=1.81$, 99\% confidence interval $(C I)=1.33$ -
\end{abstract}

This article is freely accessible online.

Correspondence to: William W Tseng, MD, Assistant Professor of Surgery, Department of Surgery, Section of Surgical Oncology, University of Southern California, Keck School of Medicine, 1510 San Pablo Street, HCC-I Suite 514, Los Angeles, CA 90033, U.S.A. Tel: +1 3238651535, Fax: +1 3238650164, e-mail: william.tseng@ med.usc.edu

Key Words: Liposarcoma, second primary neoplasms, epidemiology, soft tissue sarcoma.
2.40). Patients less than 50 years old at the time of liposarcoma diagnosis had a higher $O / E$ ratio for second primary malignancy compared to older patients. A total of 269 patients (14.6\%) developed a second cancer $(O / E=1.33$, 99\% CI=1.151.54). Conclusion: In some patients with WD/DD liposarcoma, there appears to be an increased risk of having a second primary cancer. Further validation and investigation is needed, as this finding may have implications (e.g. closer screening) for patients with this disease.

Well-differentiated/dedifferentiated (WD/DD) liposarcoma is a rare malignancy of putative adipocyte origin (1). WD/DD liposarcoma represents a disease spectrum ranging from WD tumors that are low grade and indolent to DD tumors that are high grade and can be aggressive. Both WD and DD liposarcoma exhibit a common amplification of chromosome 12q13-15, which includes genes MDM2 proto-oncogene (an inhibitor of the tumor suppressor gene p53) and cyclindependent kinase 4 (CKD4) (a critical regulator of cell cycling) (2). This is characteristic and diagnostic of the disease. There are no known risk factors (e.g. no association with any lifestyle, environmental, or socioeconomic factors) and to date, a genetic predisposition has not been reported.

Clinical management of WD/DD liposarcoma can be very challenging. These tumors tend to occur in deep-tissue spaces such as the retroperitoneum, where they can reach massive size, typically $20-30 \mathrm{~cm}$. Surgery is the mainstay of treatment; however, complete resection is difficult due to tumor size, location, and potential involvement of nearby vital organs and critical structures. WD/DD liposarcoma has a high risk of locoregional recurrence even after apparent complete resection; tumors with evidence of DD also have the potential for distant metastasis (3). To date, radiation therapy and chemotherapy have not been shown to be effective. After treatment (e.g. surgery), close surveillance 
with cross-sectional imaging is critical. Guidelines for surveillance exist for sarcoma in general, however, there are none specific for WD/DD liposarcoma.

Previous unpublished data from one of the authors (WWT) of our group suggested a higher frequency of second primary malignancies in patients with WD/DD liposarcoma. In order to assess this finding in a systematic manner, we used two datasets. Firstly, we explored the frequency of second cancer among case series of patients with WD/DD liposarcoma at three sarcoma referral centers, two in the United States (US) and one in Europe, and report the frequency of second cancer in our combined case series. Secondly, we assessed the observed versus expected occurrence of second cancer among WD/DD liposarcoma cases included in a population-based US national cancer registry database. We hypothesized that this occurrence would be more than would be expected by chance.

\section{Materials and Methods}

Consecutive cases of primary incident WD/DD liposarcoma diagnosed between 2014-2016 were combined from three sarcoma referral centers: University of Southern California, including affiliated hospitals under the Sarcoma Program in Los Angeles, CA, USA; The James Comprehensive Cancer Center of Ohio State University in Columbus, OH, USA; and the IRCCS Foundation National Tumor Institute in Milan, Italy.

Demographic and clinicopathological data were collected for patients with WD/DD liposarcoma who were found to have a second primary cancer either concurrently or within 2 years of liposarcoma diagnosis. Each center also provided the total number of consecutive patients with WD/DD liposarcoma seen during the same time period.

The United States National Cancer Institute's Surveillance, Epidemiology and End Results (SEER) database was also utilized. The SEER program is a comprehensive source of population-based cancer registry data that includes patient demographics, primary tumor site and histology, and other information provided by cancer registries across the country. All adult patients were selected from nine combined SEER registries who were diagnosed with WD/DD liposarcoma [International Classification of Diseases for Oncology, 3rd edition (4) (ICD-O-3) histology codes 8851.3 for WD and 8858.3 for DD] between 1973-2012. Myxoid liposarcoma (ICD-O-3 code 8852.3) was not included in our study. Patients diagnosed with liposarcoma not otherwise specified (ICD-O-3 code 8850.3) were also excluded to prevent other forms of liposarcoma (e.g. pleomorphic) and benign lipomas from being inadvertently included in our analysis. Using this set of nine combined registries covered approximately $9.4 \%$ of the total US population based on the 2010 census, and included the largest number of total cases of WD/DD liposarcoma in the SEER database at the time of our study, based on total years of diagnosis (5).

For the SEER database, observed/expected $(\mathrm{O} / \mathrm{E})$ ratios of concurrent or subsequent primary malignancies were calculated by comparing the number observed to the number that would have been expected based on the age-adjusted cancer incidence in the general population using the multiple primary standardized incidence ratio (MIP-SIR) session of SEER*stat software. MP-SIR is a method used to perform multiple primary analyses and to test hypotheses that explore theoretical links in the etiology of two cancers. A defined cohort of persons previously diagnosed with cancer is followed through time to compare their incidence of second primary malignancies (based on SEER coding rules) to the expected incidence of second primary malignancies in the general population. A $99 \%$ confidence interval for the $\mathrm{O} / \mathrm{E}$ ratio was calculated $(p<0.01)$, where a statistically significant confidence interval was one that excluded an $\mathrm{O} / \mathrm{E}$ ratio of 1 . Of note, the $\mathrm{O} / \mathrm{E}$ ratio measures the number of tumors, not patients. A single patient may have contributed more than one subsequent primary tumor; this distinction was noted in the results. Cases where the diagnosis of WD/DD liposarcoma was made only by death certificate or autopsy were excluded.

\section{Results}

Case series. From 2014 to 2016, from three sarcoma referral centers, there was a total of 26 out of $312(8.3 \%)$ consecutive patients with a second primary cancer either concurrent or within 2 years after WD/DD liposarcoma diagnosis. An example of one case is shown in Figure 1. The combined data including second cancer diagnoses are listed in Table I. The frequency of second cancer was $3.1 \%$ (three out of 96) at Ohio State University, 8.9\% (16 out of 178) at the Milan National Tumor Institute and 18\% (seven out of 38) at the University of Southern California.

SEER data. From the SEER database, 1,845 patients (61.0\% male) were identified with the diagnosis of WD/DD LPS between 1973 and 2012. A total of 1,345 patients $(60.0 \%$ male) were diagnosed with WD LPS, while 500 patients (63.0\% male) were diagnosed with DD LPS. Among them, 75 (4.1\%) had 80 concurrent or subsequent additional primary malignancies within 2 years (23 months) of liposarcoma diagnosis (1.47 mean person-years at risk). This represents a statistically significant $\mathrm{O} / \mathrm{E}$ ratio of 1.81 (99\% $\mathrm{CI}=1.33-2.40$, $p<0.01)$. For patients under the age of 50 years at initial diagnosis, a higher $\mathrm{O} / \mathrm{E}$ ratio of 4.94 (99\% $\mathrm{CI}=4.83-5.06$, $p<0.01)$ was observed compared to patients aged 50 years or more $(\mathrm{O} / \mathrm{E}$ ratio=1.66, 99\% $\mathrm{CI}=1.65-1.67)$. Males had a higher $\mathrm{O} / \mathrm{E}$ ratio of 6.49 for those younger than 50 years $(99 \%$ $\mathrm{CI}=6.24-6.75$ ), while females had a higher $\mathrm{O} / \mathrm{E}$ ratio of 2.02 for those aged 50 years and older (99\% CI=2.00-2.04).

A total of $269(14.6 \%)$ patients had 314 concurrent or subsequent additional primary cancer at any point following liposarcoma diagnosis. Mean person-years at risk was 6.52 . This was also statistically significant, with an $\mathrm{O} / \mathrm{E}$ ratio of $1.33(99 \% \mathrm{CI}=1.15-1.54, p<0.01)$. The $\mathrm{O} / \mathrm{E}$ ratio for the occurrence of second primary cancer for patients under the age of 50 years at initial diagnosis was 1.73 (99\% CI=1.71$1.75, p<0.01)$, while that for patients aged 50 years or more was $1.14(99 \% \mathrm{CI}=1.13-1.14, p<0.01)$. The most common locations of second cancer and associated $\mathrm{O} / \mathrm{E}$ ratios are shown in Table II. The highest $\mathrm{O} / \mathrm{E}$ ratios were associated with second cancer in the retroperitoneum, testis, soft tissues, and kidney diagnosed within 2 years of the LPS diagnosis. 

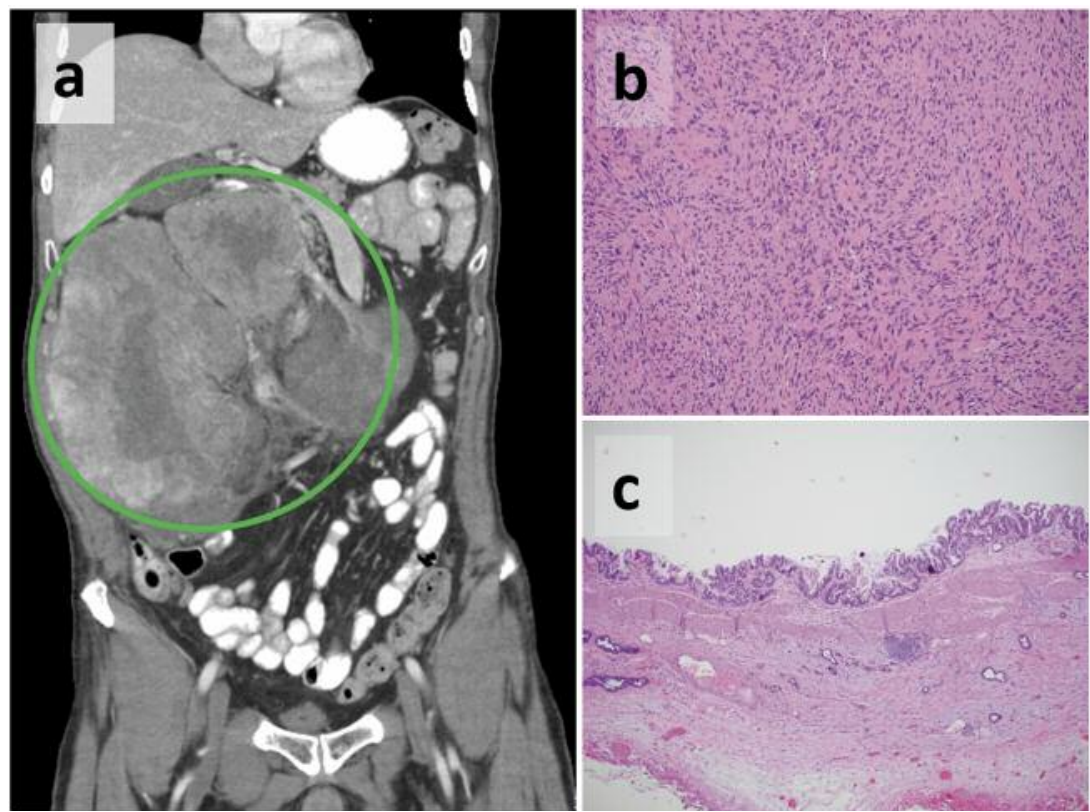

Figure 1. Example of a patient with de-differentiated liposarcoma and concurrent gall bladder adenocarcinoma. Coronal computed tomographic scan showing large liposarcoma (circled) in the right retroperitoneum (a). Histological images confirming the diagnosis of liposarcoma (b) and gallbladder adenocarcinoma $(c)$.

Table I. Combined case series data from three referral centers for patients with liposarcoma (LPS) and a second primary cancer.

\begin{tabular}{|c|c|c|c|c|c|c|}
\hline Center & $\begin{array}{l}\text { Age, } \\
\text { years }\end{array}$ & Gender & $\begin{array}{l}\text { LPS } \\
\text { subtype }\end{array}$ & $\begin{array}{l}\text { LPS } \\
\text { location }\end{array}$ & $\begin{array}{l}\text { Second primary } \\
\text { cancer diagnosis }\end{array}$ & $\begin{array}{l}\text { Time interval between } \\
\text { LPS and second cancer }\end{array}$ \\
\hline USC & 59 & M & WD & $\mathrm{RP} /$ pelvis & T-Cell lymphoma & Concurrent \\
\hline USC & 74 & M & WD & $\mathrm{RP} /$ pelvis & Prostate cancer & Concurrent \\
\hline USC & 67 & M & $\mathrm{DD}$ & Ant. abdomen & Papillary thyroid carcinoma & Concurrent \\
\hline USC & 68 & M & DD & $\mathrm{RP} /$ pelvis & Gallbladder carcinoma & Concurrent \\
\hline USC & 47 & M & DD & $\mathrm{RP} /$ pelvis & Renal cell carcinoma & 24 Months \\
\hline USC & 63 & M & DD & SBM & Sigmoid colon carcinoma & Concurrent \\
\hline USC & 58 & M & WD & $\mathrm{RP} /$ pelvis & Renal angiomyolipoma, GIST & Concurrent \\
\hline Milan & 35 & M & WD & $\mathrm{RP}$ & Pheochromocytoma & Concurrent \\
\hline Milan & 68 & $\mathrm{~F}$ & DD & $\mathrm{RP}$ & Breast cancer & Concurrent \\
\hline Milan & 80 & M & DD & $\mathrm{RP}$ & Neuroendocrine tumor & Concurrent \\
\hline Milan & 60 & $\mathrm{~F}$ & WD & $\mathrm{RP}$ & Lung cancer & \\
\hline Milan & 40 & $\mathrm{~F}$ & $\mathrm{DD}$ & $\mathrm{RP}$ & GIST & Concurrent \\
\hline Milan & 77 & M & DD & $\mathrm{RP}$ & Multiple myeloma & Concurrent \\
\hline Milan & 59 & $\mathrm{~F}$ & DD & $\mathrm{RP}$ & Granular cell tumor of the ovary & Concurrent \\
\hline Milan & 75 & M & DD & $\mathrm{RP}$ & Head and neck cancer (pharynx) & 1 Month \\
\hline Milan & 67 & $\mathrm{~F}$ & $\mathrm{DD}$ & $\mathrm{RP}$ & Lung cancer (bronchioloalveolar carcinoma) & 4 Months \\
\hline Milan & 68 & M & WD & Upper leg & Prostate cancer & Concurrent \\
\hline Milan & 71 & M & WD & $\mathrm{RP}$ & Renal cancer & Concurrent \\
\hline Milan & 63 & $\mathrm{~F}$ & DD & $\mathrm{RP}$ & PIN & Concurrent \\
\hline Milan & 68 & $\mathrm{~F}$ & WD & $\mathrm{RP}$ & PIN and renal cancer & Concurrent \\
\hline Milan & 71 & M & WD & Spermatic cord & Renal cell carcinoma & 2 Months \\
\hline Milan & 82 & $\mathrm{~F}$ & DD & $\mathrm{RP}$ & Chronic lymphocytic leukemia & Concurrent \\
\hline Milan & 54 & $\mathrm{~F}$ & $\mathrm{DD}$ & $\mathrm{RP}$ & Mesenteric non-Hodgkin lymphoma & \\
\hline Ohio & 71 & $\mathrm{~F}$ & WD & $\mathrm{RP} /$ pelvis & Follicular lymphoma & 11 Months \\
\hline Ohio & 69 & M & $\mathrm{DD}$ & $\mathrm{RP} /$ pelvis & $\begin{array}{c}\text { RP leiomyosarcoma, small bowel endocrine tumor, } \\
\text { ascending colon cancer }\end{array}$ & 22 Months, 28 months \\
\hline Ohio & 64 & M & WD & Ant. abdomen & hepatocellular cancer & Concurrent \\
\hline
\end{tabular}

M: Male, F: female, LPS: liposarcoma, WD: well-differentiated, DD: de-differentiated, RP: retroperitoneum, Ant.: anterior, SBM: small bowel mesentery, GIST: gastrointestinal stromal tumor, PIN: Pancreatic intraepithelial neoplasia; USC: University of Southern California. 
Table II. Risk of subsequent primary cancer after well-differentiated (WD)/de-differentiated, (DD) liposarcoma, SEER 1973-2012.

\begin{tabular}{|c|c|c|c|c|c|c|c|c|c|}
\hline \multirow{5}{*}{$\begin{array}{l}\text { Persons starting interval } \\
\text { Person-years in interval at risk } \\
\text { Subsequent primary cancer }\end{array}$} & \multicolumn{9}{|c|}{ Months after WD/DD liposarcoma diagnosis } \\
\hline & \multicolumn{2}{|c|}{$0-23$} & \multicolumn{2}{|c|}{$24-59$} & \multicolumn{2}{|c|}{$60+$} & \multicolumn{3}{|c|}{ Total } \\
\hline & \multicolumn{2}{|c|}{1,845} & \multicolumn{2}{|c|}{1,396} & \multicolumn{2}{|c|}{1,004} & \multicolumn{3}{|c|}{1,845} \\
\hline & & 175 & & 535 & & 022 & & 14,732 & \\
\hline & $\mathrm{O}$ & $\mathrm{O} / \mathrm{E}$ & $\mathrm{O}$ & $\mathrm{O} / \mathrm{E}$ & $\mathrm{O}$ & $\mathrm{O} / \mathrm{E}$ & $\mathrm{O}$ & $\mathrm{O} / \mathrm{E}$ & EAR \\
\hline All sites & 80 & 1.81 & 61 & 1.17 & 173 & 1.24 & 314 & 1.33 & 53.1 \\
\hline All sites excluding non-melanoma skin & 79 & 1.80 & 61 & 1.18 & 172 & 1.24 & 312 & 1.33 & 52.36 \\
\hline All solid tumors & 72 & 1.83 & 52 & 1.12 & 154 & 1.24 & 278 & 1.32 & 46.29 \\
\hline Oral cavity and pharynx & 0 & 0 & 1 & 0.79 & 3 & 0.96 & 4 & 0.72 & -1.03 \\
\hline Digestive system & 17 & 1.92 & 14 & 1.33 & 39 & 1.35 & 70 & 1.45 & 14.68 \\
\hline Esophagus & 1 & 1.84 & 0 & 0 & 5 & 2.89 & 6 & 2.06 & 2.09 \\
\hline Stomach & 3 & 3.44 & 4 & 3.83 & 3 & 1.03 & 10 & 2.07 & 3.51 \\
\hline Small intestine & 2 & 11.35 & 0 & 0 & 1 & 1.7 & 3 & 3.09 & 1.38 \\
\hline Colon, rectum and anus & 3 & 0.59 & 5 & 0.82 & 10 & 0.61 & 18 & 0.65 & -6.44 \\
\hline Liver, gallbladder, intrahepatic bile duct and other biliary & 1 & 1.12 & 0 & 0 & 2 & 0.69 & 3 & 0.62 & -1.24 \\
\hline Liver & 0 & 0 & 0 & 0 & 1 & 0.59 & 1 & 0.35 & -1.27 \\
\hline Gallbladder & 0 & 0 & 0 & 0 & 0 & 0 & 0 & 0 & -0.42 \\
\hline Intra/extrahepatic bile ducts, and other biliary & 1 & 4.15 & 0 & 0 & 1 & 1.22 & 2 & 1.48 & 0.44 \\
\hline Other biliary & 1 & 5.97 & 0 & 0 & 0 & 0 & 1 & 1.07 & 0.04 \\
\hline Pancreas & 3 & 2.51 & 1 & 0.7 & 4 & 0.98 & 8 & 1.2 & 0.89 \\
\hline Retroperitoneum & 3 & 88.00 & 4 & 103.30 & 13 & 131.70 & 20 & 116.60 & 13.46 \\
\hline Peritoneum, omentum and mesentery & 1 & 29.52 & 0 & 0 & 1 & 8.87 & 2 & 10.69 & 1.23 \\
\hline Other digestive organs & 0 & 0 & 0 & 0 & 0 & 0 & 0 & 0 & -0.2 \\
\hline Respiratory system & 8 & 1.12 & 9 & 1.06 & 26 & 1.13 & 43 & 1.11 & 2.91 \\
\hline Nose, nasal cavity and middle ear & 0 & 0 & 0 & 0 & 0 & 0 & 0 & 0 & -0.22 \\
\hline Larynx & 1 & 2.25 & 0 & 0 & 1 & 0.77 & 2 & 0.89 & -0.18 \\
\hline Pleura & 0 & 0 & 0 & 0 & 0 & 0 & 0 & 0 & -0.01 \\
\hline Lung, bronchus, trachea, mediastinum and other respiratory organs & 7 & 1.05 & 9 & 1.14 & 25 & 1.16 & 41 & 1.14 & 3.32 \\
\hline Lung and bronchus & 7 & 1.05 & 8 & 1.02 & 25 & 1.16 & 40 & 1.11 & 2.69 \\
\hline Trachea & 0 & 0 & 0 & 0 & 0 & 0 & 0 & 0 & -0.02 \\
\hline Mediastinum and other respiratory organs & 0 & 0 & 1 & 152.46 & 0 & 0 & 1 & 34.29 & 0.66 \\
\hline Bones and joints & 1 & 24.36 & 1 & 21.53 & 2 & 17.39 & 4 & 19.75 & 2.58 \\
\hline Soft tissue including heart & 3 & 14.02 & 1 & 4.01 & 14 & 20.85 & 18 & 15.86 & 11.45 \\
\hline Skin excluding basal and squamous & 5 & 2.89 & 0 & 0 & 11 & 2.1 & 16 & 1.79 & 4.78 \\
\hline Melanoma of the skin & 4 & 2.55 & 0 & 0 & 10 & 2.13 & 14 & 1.74 & 4.04 \\
\hline Other non-epithelial skin & 1 & 6.22 & 0 & 0 & 1 & 1.8 & 2 & 2.2 & 0.74 \\
\hline Breast & 3 & 0.75 & 4 & 0.85 & 10 & 0.83 & 17 & 0.82 & -2.51 \\
\hline Female breast & 3 & 0.76 & 4 & 0.87 & 9 & 0.76 & 16 & 0.79 & -2.96 \\
\hline Male breast & 0 & 0 & 0 & 0 & 1 & 4.81 & 1 & 2.88 & 0.44 \\
\hline Female genital system & 1 & 0.62 & 1 & 0.53 & 4 & 0.85 & 6 & 0.73 & -1.49 \\
\hline Cervix uteri & 0 & 0 & 0 & 0 & 0 & 0 & 0 & 0 & -0.55 \\
\hline Corpus and uterus, NOS & 1 & 1.15 & 1 & 0.99 & 2 & 0.8 & 4 & 0.91 & -0.26 \\
\hline Corpus uteri & 1 & 1.17 & 1 & 1.01 & 2 & 0.82 & 4 & 0.94 & -0.19 \\
\hline Uterus, NOS & 0 & 0 & 0 & 0 & 0 & 0 & 0 & 0 & -0.07 \\
\hline Ovary & 0 & 0 & 0 & 0 & 2 & 1.51 & 2 & 0.88 & -0.19 \\
\hline Vagina & 0 & 0 & 0 & 0 & 0 & 0 & 0 & 0 & -0.09 \\
\hline Vulva & 0 & 0 & 0 & 0 & 0 & 0 & 0 & 0 & -0.3 \\
\hline Other female genital organs & 0 & 0 & 0 & 0 & 0 & 0 & 0 & 0 & -0.1 \\
\hline Male genital system & 20 & 2.06 & 12 & 1.04 & 25 & 0.81 & 57 & 1.1 & 3.42 \\
\hline Prostate & 17 & 1.77 & 11 & 0.97 & 23 & 0.76 & 51 & 0.99 & -0.26 \\
\hline Testis & 2 & 32.01 & 0 & 0 & 0 & 0 & 2 & 8.5 & 1.2 \\
\hline Penis & 0 & 0 & 0 & 0 & 0 & 0 & 0 & 0 & -0.17 \\
\hline Other male genital organs & 1 & 58.73 & 1 & 49.9 & 2 & 36.93 & 4 & 43.85 & 2.65 \\
\hline Urinary system & 12 & 3.08 & 8 & 1.74 & 16 & 1.27 & 36 & 1.71 & 10.13 \\
\hline Urinary bladder & 5 & 1.95 & 5 & 1.65 & 8 & 0.96 & 18 & 1.29 & 2.74 \\
\hline Kidney and renal pelvis & 7 & 5.68 & 2 & 1.4 & 8 & 2.08 & 17 & 2.61 & 7.12 \\
\hline
\end{tabular}


Table II. Continued

\begin{tabular}{|c|c|c|c|c|c|c|c|c|c|}
\hline Subsequent primary cancer & $\mathrm{O}$ & $\mathrm{O} / \mathrm{E}$ & $\mathrm{O}$ & $\mathrm{O} / \mathrm{E}$ & $\mathrm{O}$ & $\mathrm{O} / \mathrm{E}$ & $\mathrm{O}$ & $\mathrm{O} / \mathrm{E}$ & EAR \\
\hline Renal pelvis, ureter and other urinary & 0 & 0 & 1 & 3.97 & 0 & 0 & 1 & 0.86 & -0.11 \\
\hline Kidney & 7 & 6.20 & 2 & 1.54 & 8 & 2.27 & 17 & 2.86 & 7.5 \\
\hline Renal pelvis & 0 & 0 & 0 & 0 & 0 & 0 & 0 & 0 & -0.38 \\
\hline Ureter & 0 & 0 & 1 & 12.17 & 0 & 0 & 1 & 2.65 & 0.42 \\
\hline Other urinary organs & 0 & 0 & 0 & 0 & 0 & 0 & 0 & 0 & -0.15 \\
\hline Eye and orbit & 0 & 0 & 0 & 0 & 1 & 5.35 & 1 & 3.06 & 0.46 \\
\hline Eye and orbit-non-melanoma & 0 & 0 & 0 & 0 & 0 & 0 & 0 & 0 & -0.04 \\
\hline Eye and orbit - melanoma & 0 & 0 & 0 & 0 & 1 & 6.65 & 1 & 3.77 & 0.5 \\
\hline Brain and other nervous system & 0 & 0 & 1 & 1.92 & 1 & 0.77 & 2 & 0.88 & -0.19 \\
\hline Brain & 0 & 0 & 1 & 2.01 & 1 & 0.8 & 2 & 0.92 & -0.12 \\
\hline Cranial nerves other nervous system & 0 & 0 & 0 & 0 & 0 & 0 & 0 & 0 & -0.07 \\
\hline Endocrine system & 3 & 6.21 & 0 & 0 & 3 & 2.32 & 6 & 2.59 & 2.5 \\
\hline Thyroid & 3 & 6.77 & 0 & 0 & 3 & 2.55 & 6 & 2.84 & 2.64 \\
\hline Thymus, adrenal gland and other endocrine & 0 & 0 & 0 & 0 & 0 & 0 & 0 & 0 & -0.14 \\
\hline All lymphatic and hematopoietic diseases & 6 & 1.64 & 8 & 1.86 & 15 & 1.27 & 29 & 1.47 & 6.29 \\
\hline Lymphoma & 4 & 2.18 & 1 & 0.47 & 5 & 0.86 & 10 & 1.02 & 0.15 \\
\hline Hodgkin lymphoma & 0 & 0 & 0 & 0 & 0 & 0 & 0 & 0 & -0.37 \\
\hline Non-Hodgkin lymphoma & 4 & 2.32 & 1 & 0.49 & 5 & 0.91 & 10 & 1.08 & 0.51 \\
\hline Nodal & 4 & 3.41 & 0 & 0 & 2 & 0.54 & 6 & 0.96 & -0.18 \\
\hline Extranodal & 0 & 0 & 1 & 1.55 & 3 & 1.68 & 4 & 1.34 & 0.69 \\
\hline Myeloma & 0 & 0 & 2 & 2.77 & 6 & 2.88 & 8 & 2.34 & 3.11 \\
\hline Leukemia & 2 & 1.66 & 5 & 3.51 & 4 & 1.02 & 11 & 1.68 & 3.03 \\
\hline Lymphocytic leukemia & 2 & 3.28 & 2 & 2.79 & 1 & 0.51 & 5 & 1.53 & 1.17 \\
\hline Acute & 0 & 0 & 0 & 0 & 0 & 0 & 0 & 0 & -0.12 \\
\hline Chronic & 2 & 3.76 & 2 & 3.2 & 1 & 0.58 & 5 & 1.73 & 1.44 \\
\hline Other & 0 & 0 & 0 & 0 & 0 & 0 & 0 & 0 & -0.15 \\
\hline Non-lymphocytic leukemia & 0 & 0 & 3 & 4.24 & 3 & 1.53 & 6 & 1.84 & 1.86 \\
\hline Acute & 0 & 0 & 2 & 4.43 & 2 & 1.6 & 4 & 1.92 & 1.3 \\
\hline Myeloid and monocytic leukemia & 0 & 0 & 3 & 4.9 & 3 & 1.78 & 6 & 2.13 & 2.16 \\
\hline Acute myeloid leukemia & 0 & 0 & 2 & 5.25 & 2 & 1.88 & 4 & 2.26 & 1.52 \\
\hline Acute monocytic leukemia & 0 & 0 & 0 & 0 & 0 & 0 & 0 & 0 & -0.07 \\
\hline Chronic myeloid leukemia & 0 & 0 & 1 & 5.44 & 1 & 1.99 & 2 & 2.37 & 0.79 \\
\hline Mesothelioma & 0 & 0 & 0 & 0 & 0 & 0 & 0 & 0 & -0.48 \\
\hline Kaposi sarcoma & 0 & 0 & 0 & 0 & 0 & 0 & 0 & 0 & -0.16 \\
\hline Miscellaneous & 1 & 1.05 & 1 & 0.86 & 3 & 0.94 & 5 & 0.94 & -0.22 \\
\hline
\end{tabular}

E: Expected number of subsequent primary cancers; O/E: ratio of observed to expected cancers; EAR: excess absolute risk per 10,000 personyears $=[(\mathrm{O}-\mathrm{E}) /$ person-years at risk $] \times 10,000$.

\section{Discussion}

In this study, we found that there was an increased risk of a second primary cancer in patients with WD/DD liposarcoma, especially among males diagnosed under 50 years of age. There are numerous previous case reports of concurrent cancer (Table III) (6-20) in WD/DD liposarcoma which add further evidence to support our observation; however, to our knowledge, the current study is the first to report data from a large case series and provide data from analysis of a national population-based cancer database to assess this occurrence.

This study raises questions regarding the etiology of second primary cancer in patients with WD/DD liposarcoma. The younger group ( $<50$ years old) in the SEER database had a higher $\mathrm{O} / \mathrm{E}$ ratio for second cancer, especially for those occurring within 2 years. WD/DD liposarcoma is characterized by 12q13-15 amplification, a hallmark genetic feature in this disease. While there are no known genetic cancer syndromes associated with 12q13-15 amplification, liposarcoma has been infrequently reported in select patients with Li-Fraumeni syndrome and neurofibromatosis $(9,21$ 23). There are no other defined genetic cancer syndromes associated with a higher risk of liposarcoma to our knowledge. Interestingly, a recent study by Ballinger et al. found that in a large combined cohort of 1,162 patients with sarcoma, using targeted exon sequencing, more than half were actually found to have an excess of pathogenic germline monogenic and polygenic variants (24). These variants were associated with earlier age of onset, and 
Table III. Case reports in the literature on well-differentiated (WD)/de-differentiated, (DD) liposarcoma (LPS) and second primary malignancies (6-20).

\begin{tabular}{|c|c|c|c|c|c|}
\hline First author (Ref) & Year & Age, years & LPS subtype & LPS location & Second primary cancer diagnosis \\
\hline Christodoulidou et al. (6) & 2015 & 60 & DD & Paratesticular & Paratesticular angiolipoma (contralateral) \\
\hline Hoshi et al. (7) & 2014 & 34 & WD* & Retroperitoneum & Clear cell renal cell carcinoma \\
\hline Frank and Velasco (8) & 2013 & 74 & DD & Retroperitoneum & Papillary cell renal cell carcinoma \\
\hline Coyle et al. (9) & 2013 & 69 & DD & Paratesticular & Prostate adenocarcinoma and unilateral renal cell carcinoma \\
\hline Saleh et al. (10) & 2013 & 62 & WD & Esophageal & Hurthle cell carcinoma of right thyroid \\
\hline Kast et al. (11) & 2012 & 40 & DD/WD & Unspecified & Myxofibrosarcoma of right arm, bilateral breast cancer \\
\hline Borriello et al. (12) & 2012 & 59 & WD/myxoid & Axilla & Breast cancer \\
\hline Demirci et al. (13) & 2010 & 69 & WD & Paratesticular & Prostate adenocarcinoma \\
\hline Kinebuchi et al. (14) & 2009 & 60 & WD & Peri-renal & Clear cell renal cell carcinoma \\
\hline Jeong et al.(15) & 2009 & 68 & WD & Buttock & Amelanotic melanoma \\
\hline Williamson et al. (16) & 2008 & 79 & WD & Retroperitoneum & Papillary cell renal cell carcinoma \\
\hline Hatano et al. (17) & 2007 & 78 & WD & Spermatic cord & Adenocarcinoma of rectum \\
\hline Hirata et al. (18) & 2006 & 40 & WD & Thigh & $\begin{array}{l}\text { Urothelial bladder cell cancer, rectal cancer. } \\
\text { MSH2 mut. in HNPCC }\end{array}$ \\
\hline Galazka et al. (19) & 2003 & 58 & DD & Cardiac (by autopsy) & Papillary cell renal cell carcinoma \\
\hline Lewis et al. (20) & 1994 & 71 & WD & Perirenal & Granular cell renal cell carcinoma \\
\hline
\end{tabular}

MSH2 mut.: Mutation in DNA mismatch repair protein Msh2; HNPCC: hereditary nonpolyposis colorectal cancer. *WD on pathology, myxoid on magnetic resonance imaging.

suggested that a large percentage of sarcomas may in fact have some underlying hereditary and genetic component. These findings were supported by Chan et al. in a similar study of an Asian cohort of patients with sarcoma, which found that $13.6 \%$ of patients with sporadic sarcomas exhibited at least one pathogenic germline mutation in 10 cancer-associated genes (25).

It is also possible that the occurrence of a second cancer in patients with WD/DD liposarcoma may have another etiology. The amplified locus at 12q13-15 contains several genes overexpression of which may account for the development of some of the secondary cancers. Alternatively, the presence of liposarcoma, especially given its often large tumor size, may induce systemic immune responses (e.g. relative immunosuppression) that predisposes the patient to the development of a second cancer. Interestingly, both the current SEER*stat analysis (Table II) and case reports in the literature (Table III) show that these second primary cancers often occur in the genitourinary system. Specifically, concurrent renal cell carcinoma is frequently observed. While this may be a sampling bias as the ipsilateral kidney is often removed along with retroperitoneal liposarcoma during surgery, this could also be due to local immune responses in the kidney being shifted to a pro-tumorigenic bias due to the large, adjacent liposarcoma. These hypotheses deserve further study, and may also be relevant to other, more common cancer types.

The true frequency of a second primary cancer in WD/DD liposarcoma is currently unknown and cannot be definitively derived from this study. In general, it is recognized that all patients with cancer, regardless of cancer site, have a somewhat increased risk of developing another primary cancer during their lifetime. In reported epidemiological studies, this frequency ranges from $1 \%$ for liver cancer up to $16 \%$ for bladder cancer $(26,27)$. One study found a cumulative incidence of second cancer in all cancer survivors (of any cancer type) to be $5.0 \%$ at 5 years and up to $13.7 \%$ at 25 years. Our analysis of the SEER database suggests that the lifetime risk of developing a second cancer in patients with WD/DD liposarcoma (14.6\%) is in concordance with these prior reports and positions these patients at or beyond the higher end of this frequency range. Interestingly, a posthoc survey of a large online patient support group for liposarcoma ("Liposarcoma Survivors" on Facebook) was performed from Feb-Aug 2017. Among 134 members with WD/DD liposarcoma that responded to the survey, 26 (19\%) reported responded "yes" to ever having a history of a second cancer. Ten of these patients ( $8 \%$ total) reported the identification of the second non-liposarcoma cancer concurrently or within 2 years of the liposarcoma diagnosis, similar to the results in this study.

The true timing of a second primary cancer in relation to WD/DD liposarcoma is also unclear. Liposarcomas are usually very large at the time of detection, with a typical size of 20$30 \mathrm{~cm}$. In fact, these are probably the largest tumors in the human body (see Figure 1) (28). The growth rate and progression of liposarcoma prior to detection is poorly defined, and therefore it is possible that these tumors may have been present in a smaller, clinically occult form while the other cancer was detected (and even treated) or vice versa. 
Overall, the findings reported in the current study are intended to increase awareness of this occurrence and stimulate deeper investigation through research into this rare disease. Clinically, closer screening for the possibility of a second cancer should be incorporated into the management of patients with WD/DD liposarcoma. Ultimately, if our observations are validated, surveillance algorithms may need to be specifically modified to incorporate this risk.

\section{Conclusion}

In this study, we observed that there was an increased risk of second malignancy in some patients with WD/DD liposarcoma, especially those diagnosed under 50 years of age. These findings are based on data from a combined case series of patients with sarcoma and supported by analysis of a national population-based cancer database. Further validation is needed and laboratory-based investigation is warranted to provide insight into etiology.

\section{Disclosures}

This work had no specific funding. The Authors declare that they have no conflict of interest in regard to this study.

\section{References}

1 Dei Tos AP and Pedeutour F: Well-differentiated liposarcoma. In: Fletcher CDM, Bridge JA, Hogendoorn PCW and Mertens F (eds): WHO Classification of Tumors of Soft Tissue and Bone, Fourth Edition. International Agency for Research on Cancer (IARC): Lyon, France, pp. 33-36, 2013.

2 Tseng WW, Somaiah N, Lazar AJ, Lev DC and Pollock RE: Novel systemic therapies in advanced liposarcoma: a review of recent clinical trial results. Cancers 5(2): 529-549, 2013.

3 Vijay A and Ram L: Retroperitoneal liposarcoma: a comprehensive review. Am J Clin Oncol 38(2): 213-219, 2015.

4 International Classification of Diseases for Oncology, Third Edition, First Revision. Geneva: World Health Organization, 2013.

5 Surveillance, Epidemiology, and End Results (SEER) Program. Surveillance, Epidemiology, and End Results (SEER) Program. SEER*Stat Database: Incidence-SEER 18 Regs Research Data + Hurricane Katrina Impacted Louisiana Cases, 2012. https://seer.cancer.gov/data-software/documentation/seerstat/ nov2012/

6 Christodoulidou M, Khetrapal P, Edmunds L and Muneer A: Paratesticular liposarcoma and contralateral angiolipoma in a 60year-old patient. BMJ Case Rep 10: 1136, 2015.

7 Hoshi S, Hayashi N, Yagi M, Ookubo T, Muto A, Sugano O, Numahata K, Bilim V, Hoshi K, Sasagawa I and Saso S: Long term survival in a case of concurrent retroperitoneal liposarcoma and renal cell carcinoma: a case report. BMC Res Notes 7: 538, 2014.

8 Frank RM and Velasco JM: Surgical management of incidental renal tumor during excision of retroperitoneal liposarcoma and osteogenic sarcoma. Am Surg 79(2): 88, 2013.
9 Coyle D, Flaherty RA, Kelly BD, Hynes SO, Colesky F, Quinlan MR, Corcoran MO and Rogers E: Recurrent dedifferentiated paratesticular liposarcoma with synchronous renal cell carcinoma and prostate cancer. Curr Urol 6(4): 216218, 2013.

10 Saleh WN, Aljehani YM and Ahmad MH: Giant pedunculated esophageal liposarcoma associated with Hurthle cell thyroid neoplasia. Saudi Med J 34(7): 750-752, 2013.

11 Kast K, Krause M, Schuler M, Friedrich K, Thamm B, Bier A, Distler W and Kruger S: Late onset Li-Fraumeni Syndrome with bilateral breast cancer and other malignancies: case report and review of the literature. BMC Cancer 12: 217, 2012.

12 Borriello M, Lucidi A, Carbone A, Iannone V and Ferrandina G: Malignant transformation of Madelung's disease in a patient with a coincidental diagnosis of breast cancer: a case report. Diagn Pathol 7: 116, 2012.

13 Demirci U, Buyukberber S, Cakir A, Ozturk B, Akyurek N, Unver B, Baykara M, Benekli M and Coskun U: Synchronous testicular liposarcoma and prostate adenocarcinoma: a case report. Cases J 3: 27, 2010.

14 Kinebuchi Y, Ishizuka O, Minagawa T, Nisizawa O and Shimojo $\mathrm{H}$ : Concurrent perirenal liposarcoma associated with renal cell carcinoma. Hinyokika Kiyo 55(9): 571-574, 2009.

15 Jeong TJ, Lee EJ, Haw S, Shin MK and Haw CR: Coexistence of amelanotic melanoma and liposarcoma. Ann Dermatol 21(4): 409-412, 2009.

16 Williamson JM, Konig TC and Canelo R: Incidental finding of renal cell carcinoma in recurrent retroperitoneal liposarcoma. Ann R Coll Surg Engl 90(1): 4, 2008.

17 Hatano K, Sato M, Tsujimoto Y, Takada T, Honda M, Matsumiya K, Fujioka H, Uchikoshi F, Nakahara M, Matsuura N and Tsujimoto M: Liposarcoma of the spermatic cord associated with rectum cancer: A case report. Hinyokika Kiyo 53(8): 597-600, 2007.

18 Hirata K, Kanemitsu S, Nakayama Y, Nagata N, Itoh H, Ohnishi H, Ishikawa H, Furukawa Y, for HNPCC Registry and Genetic Testing Project of the Japanese Society for Cancer of the Colon and Rectum (JSCCR): A novel germline mutation of $\mathrm{MSH} 2$ in a hereditary nonpolyposis colorectal cancer patient with liposarcoma. Am J Gastroenterol 101(1): 193-196, 2006.

19 Galazka K, Ciezarek M, Soja J, Krzanowski M, Szlubowski A, Sydor K, Adamczyk W, Grodecki J and Sladek K: Synchronous primary heart liposarcoma and papillary renal carcinoma - a case report. Pol J Pathol 54(2): 153-159, 2003.

20 Lewis DJ, Moul JW, Williams SC, Sesterhenn IA and Colon E: Perirenal liposarcoma containing extramedullary hematopoiesis associated with renal cell carcinoma. Urology 43(1): 106-109, 1994.

21 James AW, Chang L, Genshaft S and Dry SM: Coincident liposarcoma, carcinoid and gastrointestinal stromal tumor complicating type 1 neurofibromatosis: Case report and literature review. J Orthop 12(Suppl 1): 111, 2014.

22 Gomez CK, Rosen G, Mitnick R and Chaudhri A: Recurrent retroperitoneal liposarcoma in a patient with neurofibromatosis type I: BMJ Case Rep 2012: 006310, 2012.

23 Schofer MD, Abu-Safieh MY and Paletta J, Fuchs-Winkelmann $\mathrm{S}$ and El-Zayat BF: Liposarcoma of the forearm in a man with type 1 neurofibromatosis: a case report. J Med Case Rep 3: $7071,2009$. 
24 Ballinger ML, Goode DL, Ray-Coquard I, James PA, Mitchell G, Niedermayr E, Puri A, Schiffman JD, Dite GS, Cipponi A, Maki RG, Brohl AS, Myklebost O, Stratford EW, Lorenz S, Ahn SM, Ahn JH, Kim JE, Shanley S, Beshay V, Randall RL, Judson I, Seddon B, Campbell IG, Young MA, Sarin R, BLay JY, O'Donoghue SI and Thomas DM: Monogenic and polygenic determinants of sarcoma risk: an international genetic study. Lancet Oncol 17(9): 1261-1271, 2016.

25 Chan SH, Lim WK, Ishak NDB, Li ST, Goh WL, Tan GS, Lim KH, Teo M, Young CNC, Malik S, Tan MH, Teh JYH, Chin FKC, Kesavan S, Selvarajan S, Tan P, The BT, Soo KC, Farid $\mathrm{M}$, Quek R and Ngeow J: Germline mutations in cancer predisposition genes are frequent in sporadic sarcomas. Sci Rep 7(1): 10660, 2017.

26 Hayat MJ, Howlader N, Reichman ME and Edwards BK: Cancer statistics, trends, and multiple primary cancer analyses from the Surveillance, Epidemiology, and End Results (SEER) program. Oncologist 12(1): 20-37, 2007.
27 Ye Y, Neil AL, Wills KE and Venn AJ: Temporal trends in the risk of developing multiple primary cancers: A systematic review. BMC Cancer 16(1): 849, 2016.

28 Fabre-Guillevin E, Coindre JM, Somerhausen Nde S, Bonichon F, Stoeckle E and Bui NB: Retroperitoneal liposarcomas: followup analysis of dedifferentiation after clinicopathologic reexamination of 86 liposarcomas and malignant fibrous histiocytomas. Cancer 106(12): 2725-2733, 2006.

Received March 28, 2018 Revised April 25, 2018 Accepted May 3, 2018 